\title{
Biodegradable conductive composites of poly(3-hydroxybutyrate) and polyaniline nanofibers: Preparation, characterization and radiolytic effects
}

\author{
P. L. B. Araujo ${ }^{1,2}$, C. R. P. C. Ferreira 2 , E. S. Araujo ${ }^{2}$ \\ ${ }^{1}$ Laboratório de Química de Produtos Naturais Bioativos, Departamento de Química, Universidade Federal Rural de \\ Pernambuco, Av. D. Manoel de Medeiros, s/n., 52171-900, Dois Irmãos, Recife, Brazil \\ ${ }^{2}$ Laboratório de Polímeros e Nanoestruturas, Departamento de Energia Nuclear, Universidade Federal de Pernambuco, \\ Av Prof. Luis Freire, n. 1000, 50740-540, Cidade Universitária, Recife, Brazil
}

\begin{abstract}
Poly(3-hydroxybutyrate) is a biodegradable polyester produced by microorganisms under nutrient limitation conditions. We obtained a biodegradable poly(3-hydroxybutyrate) composite having 8 to 55\% of chemically in situ polymerized hydrochloric acid-doped polyaniline nanofibers (70-100 nm in diameter). Fourier transform infrared spectroscopy and X-rays diffractometry data did not show evidence of significant interaction between the two components of the nanocomposite, and polyaniline semiconductivity was preserved in all studied compositions. Gamma-irradiation at $25 \mathrm{kGy}$ absorbed dose on the semiconductive composite presenting $28 \%$ of doped polyaniline increased its conductivity from 4.6 $10^{-2}$ to $1.1 \mathrm{~S} / \mathrm{m}$, while slightly decreasing its biodegradability. PANI-HCl biodegradation is negligible when compared to PHB biodegradability in an 80 day timeframe. Thus, this unprecedented all-polymer nanocomposite presents, at the same time, semiconductivity and biodegradability and was proven to maintain these properties after gamma irradiation. This new material has many potential applications in biological science, engineering, and medicine.
\end{abstract}

Keywords: nanocomposites, gamma-irradiation, biodegradable polymers, polyaniline, poly(hydroxybutyrate)

\section{Introduction}

Biodegradable polymers are defined as macromolecules in which the primary degradation mechanism is through the action and metabolism of microorganisms [1]. In general, biodegradable polymer materials are degraded into biomass, carbon dioxide, and/or methane. Thus, the macromolecular backbone suffers breakdown and is used as a source of carbon and energy.

A major forefront for the application of biodegradable polymers is in medical science and technology. They are used as temporary substitutes for natural tissues and degrade in vivo over a predetermined period of time generating safe end products. Among these materials, polyhydroxyalkanoates (PHAs) [2] along with poly $(\alpha$-hydroxy acids) [3] are the most used biodegradable polymers.

PHAs are polyesters produced by microorganisms under nutrient limitation conditions [4]. Poly(3hydroxybutyrate) (PHB) and copolymers of 3hydroxybutyrate and 3-hydroxyvalerate (PHB-coHV) can be considered as the most known PHAs. They present biodegradability and good biocompatibility and are frequently used, as neat substances or composites, for fabrication of medical supplies, including: sutures, screws, bone plates, orthopedic pins, guided tissue repair/regeneration devices, nerve guides, vein valves, bone marrow scaffolds,

*Corresponding author: elmo@pq.cnpq.br

(c) BME-PT 
ocular cell implants, substitutes for skin and dura tissue, wound dressing, and hemostats [2]. PHB-co$\mathrm{HV}$ can also be used as biodegradable drug carriers in implants loaded with therapeutic substances such as antibiotics [5] or anticancer drugs [6]. Among all PHAs, PHB appears to have a broader range of applications due to its good combination of mechanical, biological, and surface properties [7].

The previously-cited PHB characteristics turn this polymer into a very interesting matrix for the fabrication of multifunctional materials, namely polymer nanocomposites. In spite of their innumerous potential applications, scientific information on nanocomposites based on PHB and other PHAs are rather limited. Reports on the fabrication of PHB [8] or PHB-co-HV $[8,9]$ composites through melting intercalation showed that improved thermal tensile properties could be achieved in composites presenting nanodispersions of organoclay on PHBco-HV matrix, although, thermal degradation of PHB after melting process might have counterbalanced the improving effect of this filler on the nanocomposite [9]. Nanocomposites of PHB-co$\mathrm{HV} /$ multiwalled carbon nanotubes are reported to exhibit higher thermal stability than PHB-co-HV itself [10].

In the present work, we report the development of a simple, straightforward method to fabricate $\mathrm{PHB} /$ polyaniline (PANI) nanocomposites. PANI is an intrinsically conductive polymer (ICP) which has been extensively studied over the last twenty years, in both theoretical $[11,12]$ and practical points-ofview $[13,14]$. This polymer combines striking properties such as metal-like characteristics [15], reversible doping level [16], and good biocompatibility [17]. PANI presents four oxidation states, which can arranged in crescent order of oxidation level as leucoemeraldine, emeraldine, nigraniline and pernigraniline. Each one of these substances presents a base and a salt form [18]. The electrically conductive form of PANI is the emeraldine salt, shown in Figure 1. Such versatile polymer attracts researchers from a number of areas, leading to fre-

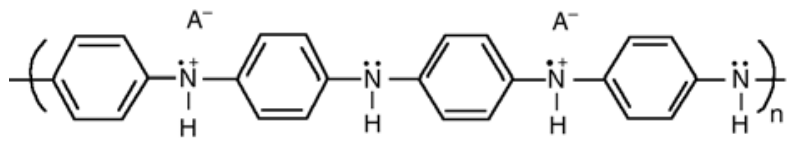

Figure 1. Polyaniline emeraldine salt, an electrically conducting form of polyaniline. $\mathrm{A}^{-}$is a counter-ion. quent reports on PANI practical uses. In recent years, our research group reported a new application of PANI as a radiostabilizing agent in nanocomposites of poly(methyl methacrylate) (PMMA) and PANI nanofibers submitted to radiosterilization $[19,20]$.

The composites presented in this work have, at the same time, biodegradability and semiconductivity and are made of in situ chemically polymerized $\mathrm{HCl}$-doped polyaniline (PANI) nanofibers as fillers and the bacterially-produced Brazilian commercial $\mathrm{PHB}$ as the matrix.

Our methodology of choice for the preparation of nanofibers embedded into PHB matrix is based on the rapid mixing of the oxidant agent with an initial short period of stirring, an approach firstly presented by Huang and Kaner, in 2006 [21], to produce bulky quantities of PANI nanofibers in water and other solvents. Following the addition of the agent, fast consumption of the oxidant occurs, and polymer chain overgrowth is prevented. In such conditions, PANI nanofibers are allowed to form, as nanostructures of this particular morphology appear to be intrinsic to PANI and other ICPs [21, 22]. Previous publications on in situ polymerization of PANI in polymer matrix emulsion were based on traditional slow, dropwise addition of reagents and continuous stirring $[23,24]$, which frequently results in irregular PANI agglomerates. [21, 25]. In order to produce PHB/PANI nanofiber composites, we performed aniline (Ani) polymerization reaction in the presence of emulsion of PHB in water/chloroform/SDS. In 2007, Ali and co-workers [26] prepared semiconducting PANI/polyvinyl alcohol (PVA) composites containing fibrous nanoclusters and aggregates of PANI, by irradiating a solution of $\mathrm{HCl}$, Ani and PVA with gamma rays up to $50 \mathrm{kGy}$. Recently, some nanofiber composites of PHB and PANI were fabricated by electrospinning method [27]. However, to our best knowledge, in situ chemical polymerization of PANI resulting in nanofibers embedded into polymer matrix was not reported, so far. In this work, we obtained good quality HCldoped PANI nanofibers/PHB composites presenting semiconductivity and biodegradability. Thus, a new methodology is suggested for the manufacturing of a polymer matrix nanocomposite.

PHB/PANI nanocomposites were gamma-irradiated at $25 \mathrm{kGy}$ dose, the standard sterilization absorbed 
dose for medical supplies, in order to assess the effects of such procedure on its electrical conductivity and biodegradability. Our results demonstrate that gamma-irradiation improved electrical conductivity while presenting little influence on the biodegradability of the nanocomposite.

Many uses of this new material can be possible, e.g., as supports for electrically stimulated/guided cell growth, similarly to what is proposed for other PANI/biodegradable polymer nanocomposites $[28,29]$.

\section{Experimental}

Aniline monomer (Ani), ammonium peroxydisulfate (APS) and ammonium hydroxyde $\left(\mathrm{NH}_{4} \mathrm{OH}\right)$ (Vetec, Rio de Janeiro, Brazil), methanol, chloroform and hydrochloric acid ( $\mathrm{HCl}$ ) (Dinâmica, Londrina, Brazil), and sodium dodecylsulfate 99\% (SDS) (Sigma-Aldrich, Saint Louis, USA) were of analytical grade. Ani was treated with stannous chloride for $24 \mathrm{~h}$ and vacuum distilled prior to use. Chloroform was dried and distilled. Commercial PHB (BIOCYCLE ${ }^{\circledR}, M_{\mathrm{w}} 530 \mathrm{~kg} / \mathrm{mol}$, PHB Industrial S. A., Usina da Pedra, Brazil) was purified by extraction with methanol in a Soxhlet apparatus for $48 \mathrm{~h}$ and air dried before use. Other chemicals were used as received.

A typical procedure for the preparation of PHB/ PANI nanocomposites was based on Ruckestein and Yang [23]: a solution of $0.2 \mathrm{~g}$ of SDS in $2 \mathrm{ml}$ of distilled deionized water was placed in a $50 \mathrm{ml}$ Becker flask. To a $5 \mathrm{ml}$ chloroform solution containing $0.4 \mathrm{~g}$ of $\mathrm{PHB}$, previously dissolved under reflux for 24 hours, was added $0.12 \mathrm{~g}$ of Ani, thus Ani to PHB ratio in mass (Ani:PHB) equaled 0.3. The resulting mixture was added to the aqueous solution under intense magnetic stirring, until forming an emulsion. A solution of $0.19 \mathrm{~g}$ of the oxidant agent, APS, in $10 \mathrm{ml}$ of $\mathrm{HCl} 1 \mathrm{~mol} \cdot \mathrm{dm}^{-3}$ was then placed at once in the emulsion and the stirring was kept working just long enough to allow an homogeneous mixing of the solutions and until an initial changing in the color of the system was perceptible (approximately $40 \mathrm{~s}$ ). The reaction was left to proceed for 3 hours at room temperature, then quenched with methanol. The precipitate formed was filtered in an Hirsch funnel, washed with methanol followed by water and $\mathrm{HCl} 1 \mathrm{~mol} \cdot \mathrm{dm}^{-3}$. The green coarse powder obtained was dried at room tempera- ture in a desiccator until constant weight and named as composite I. Similar procedure was followed to produce composites having initial Ani:PHB of 0.75 , 1.0 and 1.5, keeping all components but Ani in the same proportion (composites I, III and IV, respectively). For comparison, PANI was synthesized in the absence of PHB in the above-described conditions. For the same reason, the PHB chloroform solution was submitted to the emulsification process in the presence of APS and SDS and left to stand for 3 hours before precipitation of the polymer with methanol. Yields were calculated considering $100 \%$ as the sum of Ani, PHB and SDS weights in the reactional media [23, 24]. Samples were prepared in duplicate. PANI content in each polymer was determined gravimetrically by extraction of the soluble PHB matrix in a Soxhlet extractor with chloroform. Residual insoluble doped PANI was dried in desiccator until constant weight. Gamma irradiation of powder samples were done in air, at room temperature, in a Gammacell irradiator (220 SN 65R Source Model Number: C-198 MDS Nordion Inc, Kanata, Canada) at $25 \mathrm{kGy}$ dose and $2.61 \mathrm{~Gy} \cdot \mathrm{s}^{-1}$ dose rate.

Room temperature electrical conductivity measurements were performed in an eletrometer Keithley (model 617 Keitley Instruments Inc. Cleveland, USA) by the two-probe method on pellets of power samples pressed at $3 \mathrm{MPa}$ for $30 \mathrm{~s}$. Samples were left to rest for $72 \mathrm{~h}$ before the readings. The morphologies of the composites were investigated by scanning electron microscopy (SEM) (JEOL JSM5900, Tokyo, Japan) on gold-coated samples. Fourier Transform Infrared spectrometry (FTIR) experiments were performed on $\mathrm{KBr}$ pellets (Bruker IFS66, Ettlinger, Germany). The X-ray diffractograms (Rigaku D/max-2200, Texas, USA) were taken with $\mathrm{CuK}_{\alpha}$ radiation, $1.54 \AA, 40 \mathrm{kV}, 20 \mathrm{~mA}$, in the range of diffraction angle $2 \theta=5-35^{\circ}$ in a continuous scanning type at $1.2^{\circ}$ per min. The background and the amorphous halo were subtracted according to the methodology established by Ruland [30]. Biodegradability determination followed modified Sturm's test conditions [31], according to ASTM D5338-98 [32]. $0.8 \mathrm{~g}$ of the samples and $100 \mathrm{~g}$ of inoculant medium (worm humus, Gnumus, Vitória de Santo Antão, Brazil, 50\% of dry solids) mixed in $200 \mathrm{ml}$ of distilled water were used in each experiment. Measurements were interrupted 
when $\mathrm{CO}_{2}$ production remained approximately the same as the control sample for at least ten days. The accumulation of $\mathrm{CO}_{2}(P)$ was calculated according to the Equation (1):

$P=\sum_{n=1}^{i} \frac{m_{\mathrm{ai}}-m_{\mathrm{ci}}}{m_{\mathrm{ci}}}$

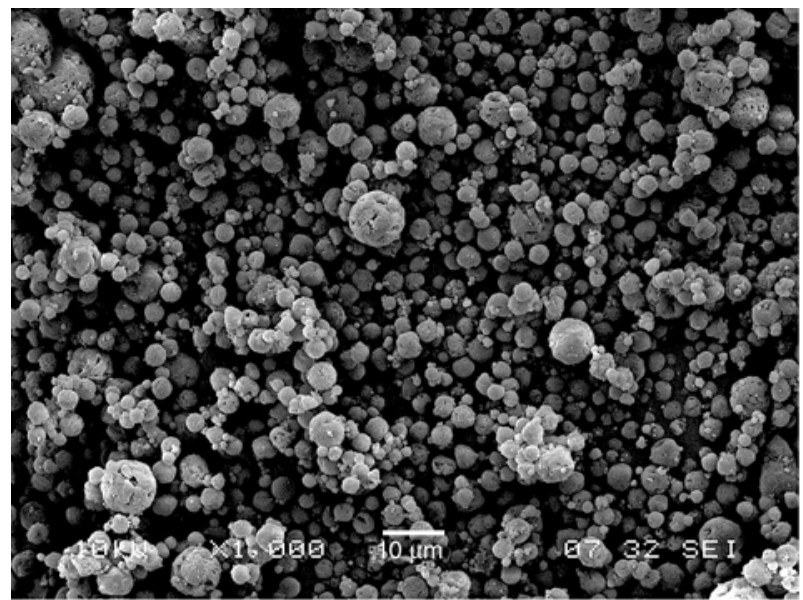

a)

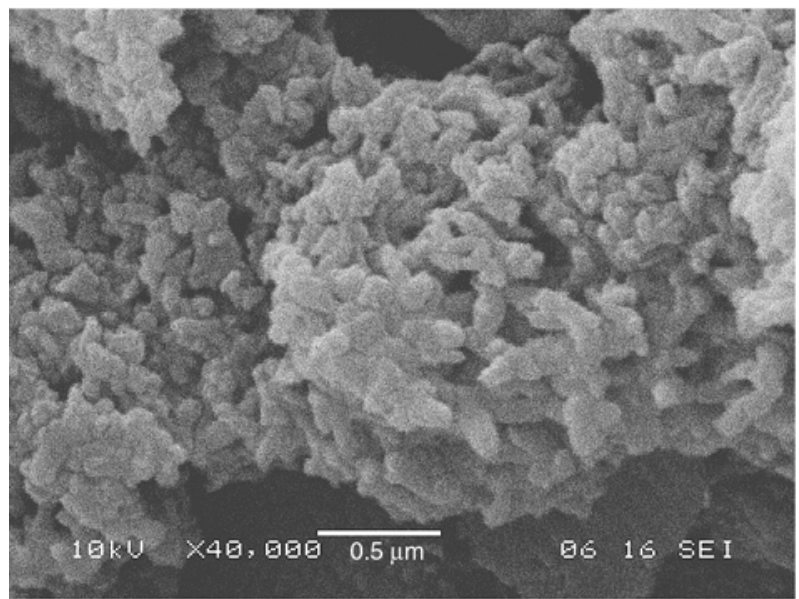

c)

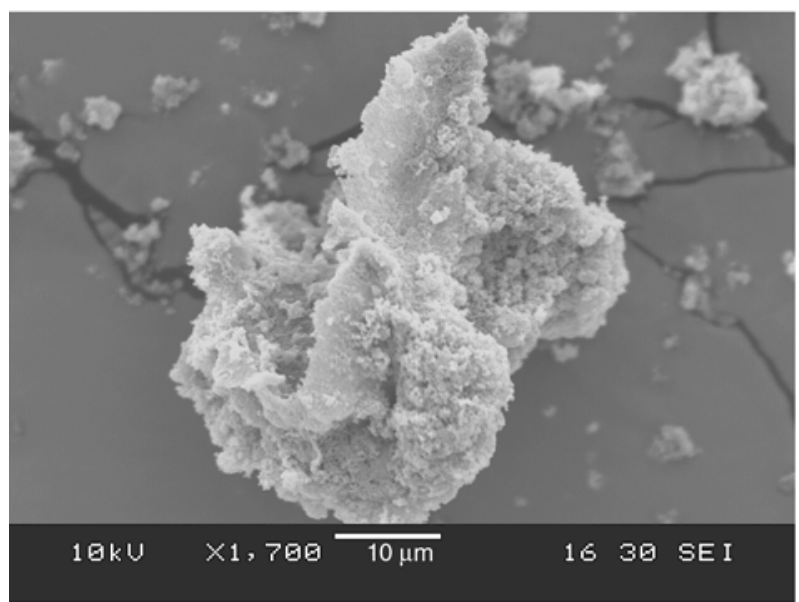

e) where $n$-number of days, $m_{\mathrm{ai}}-$ mass of $\mathrm{CO}_{2}$ produced by a sample in the $i$ day, $m_{\mathrm{ci}}-$ mass of $\mathrm{CO}_{2}$ produced by the control sample in the $i$ day.

Values were corrected to take in account only PHB percentage in the total sample mass.

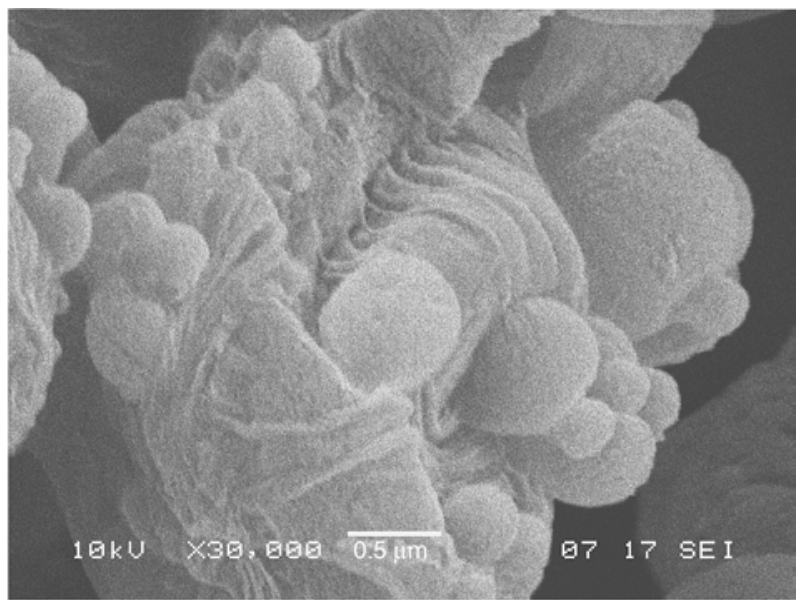

b)

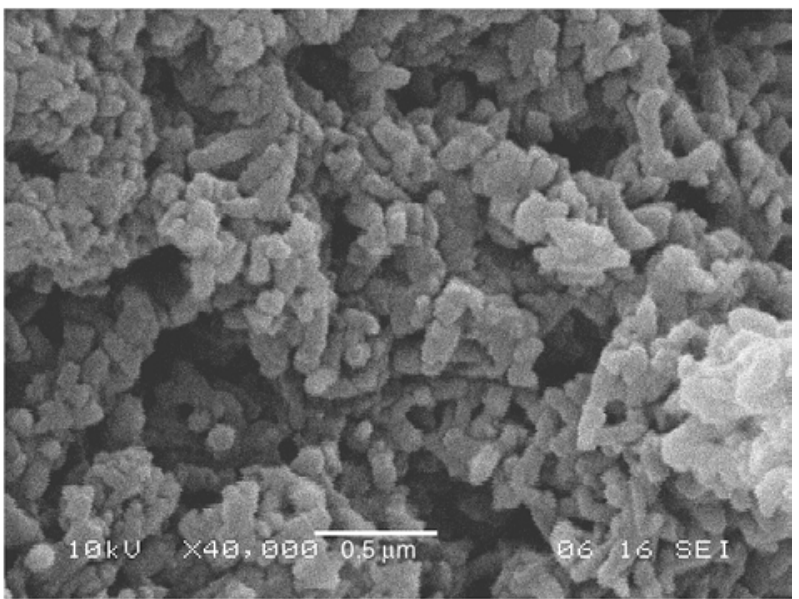

d)

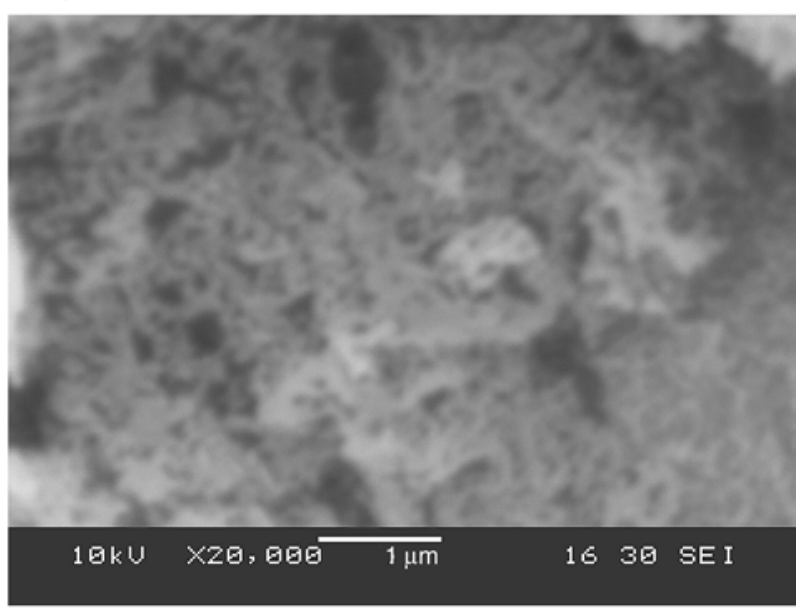

f)

Figure 2. SEM images of neat PHB (a) $\times 1000$, (b) $\times 30000$; PHB/PANI-HCl (c) nanocomposite II, nanocomposite IV. Residual PANI-HCl salt after extraction of PHB fraction with chloroform (e) $\times 1700$ and (f) $\times 20000$. 


\section{Results and discussion}

\subsection{Morphology of PHB and PHB/PANI composites}

Scanning electron micrographs of treated PHB samples exhibited spherical granules ranging from $10 \mu \mathrm{m}$ (Figure 2a) to $260 \mathrm{~nm}$ (Figure 2b) in diameter. Granules of PHB found in the cytoplasm of the bacteria Alcaligenes eutrophus, the microorganism used in the production of Brazilian PHB, presented diameters from 200 to $500 \mathrm{~nm}$ [33]. Larger granular structures may be formed during extraction process. Composites of PHB and HCl-doped PANI (PHB/ PANI-HCl) presented distinct morphologies from the neat PHB. Figures $2 \mathrm{c}$ and $2 \mathrm{~d}$ show electron micrograph images from composites II and IV where fibers or rods with diameters around $70-100 \mathrm{~nm}$ are visualized. After extraction of PHB with chloroform, residual PANI-HCl has a sponge-like appearance (Figure 2e). A network of fibrils can be identified from a higher magnification image (Figure 2f). Images of composite I did not allow a clear visualization of PANI nanostructures. These results characterize formation and good dispersion of PANI$\mathrm{HCl}$ nanofibers into the $\mathrm{PHB}$ matrix when polymerized in situ with rapid mixing of APS.

Previous works on PANI composites fabricated by in situ emulsion polymerization with polystyrene [23] and PMMA [24] used traditional slow addition of reagents during PANI synthesis. Information on the morphology of PANI structures and the methodology for calculating its content in the composite were not given at that time. At the present it is difficult to infer about the role of each emulsion component on the final morphology of PANI when rapid mixing is performed. In one hand, intrinsic fibrillar morphology is reported to be prevalent in both aqueous and organic media [21]. Moreover, nanofibrillar morphology is also observed when PANI synthesis occurs at the interface of water/immiscible organic solvent biphasic systems [21, 34]. Hence, it is arguable the formation of such structures regardless the existence of two solutions of immiscible solvents in an emulsionated media, which is the environment found in our experiment. On the other hand, we used SDS, a surfactant agent to promote emulsification. Since surfactant agents are known to act as soft templates for PANI nanofibers fabrication [35, 36], the SDS influence on nanofiber formation cannot be ruled out.

Our present approach appears to be very promising for the fabrication of fibrilar PANI nanocomposites, because it precludes the use of hard templates, electrospinning apparatus, or ionizing radiation sources in order to obtain nanofibers, and, at the same time, makes possible to embed inexpensive, insoluble, self-assembled HCl-doped PANI nanofibers directly into host polymer matrix.

\subsection{Yields, PANI content and electrical conductivity of the PHB/PANI-HCI nanocomposites}

Composites obtained as described in the previous section were analyzed for yields, PANI content, and conductivity. Results are shown in Table 1. Nanocomposite I presented the highest yield among the studied materials, followed by nanocomposite IV. Neat PHB conductivity was in the range of $10^{-12} \mathrm{~S} / \mathrm{m}$, while all nanocomposite materials obtained were above the electrical percolation threshold and presented semiconductivity. Since PHB is an electrical insulator, conductivity presented by the composites is due to PANI-HCl. For nanocomposites I-III, conductivities were situated in the same order of magnitude, around $1.0 \cdot 10^{-2} \mathrm{~S} / \mathrm{m}$. PANI pristine polymerized in the emulsion water/chloroform had a conductivity of $1.2 \mathrm{~S} / \mathrm{m}$ which is close to the conductivity reached by composite IV, $7.0 \cdot 10^{-1} \mathrm{~S} / \mathrm{m}$. Fluctuations in conductivity of PANI and its composites prepared under controlled conditions may be attributed to a large number of factors, including the presence of humidity [16], morphology and the dispersion of the filler in the composite [37]. Thus, all the samples presented comparable conductivities and are suitable candidates for applications as semiconducting biomaterials.

Table 1. Yields, PANI-HCl contents, and conductivity of PHB/PANI-HCl composites

\begin{tabular}{|c|c|c|c|c|c|}
\hline $\begin{array}{c}\text { Composite } \\
\text { PHB/PANI }\end{array}$ & $\begin{array}{c}\text { Ani/PHB } \\
\text { ratio }\end{array}$ & $\begin{array}{c}\text { Yield of PHB/PANI } \\
{[\mathbf{\%}]}\end{array}$ & $\begin{array}{c}\text { PANI-HCI } \\
{[\%]}\end{array}$ & $\begin{array}{c}\text { PANI-HCI/Ani } \\
{[\%]}\end{array}$ & $\begin{array}{c}\text { Conductivity } \\
{[\mathbf{\%} / \mathbf{m}]}\end{array}$ \\
\hline I & 0.30 & 93 & 8 & 43 & $2.1 \cdot 10^{-2}$ \\
\hline II & 0.75 & 51 & 28 & 45 & $4.6 \cdot 10^{-2}$ \\
\hline III & 1.00 & 46 & 55 & 64 & $1.8 \cdot 10^{-2}$ \\
\hline IV & 1.50 & 71 & 48 & 68 & $7.0^{-2} \cdot 10^{-1}$ \\
\hline
\end{tabular}


As composite II presents good conductivity and the lower PANI-HCl content in which nanofibers could be visualized, it was chosen for experiments of gamma irradiation and biodegradation. After exposed to a $25 \mathrm{kGy}$ absorbed dose, conductivity of nanocomposite II increased in two orders of magnitude and reached $1.1 \mathrm{~S} / \mathrm{m}$. Hence, nanocomposite semiconductivity was maintained after irradiation procedure. This result opens up an opportunity for the fabrication of gamma rays-sterilizable, biodegradable, electroactive supports for a number of biotechnology applications.

Very little information is available on conductivity behavior of chemically synthesized PANI exposed to gamma-rays. A direct comparison with previous published results is rather difficult, since most reports are based on conductivity onset observed when gamma radiation induces doping process in insulator PANI emeraldine base, resulting in semiconducting PANI forms, either in composites [3841] or pristine PANI films [42]. Thus, conductivity increases observed were of several orders of magnitude.

Our material presented semiconducting filler content above the electrical percolation limit, hence radiation-induced conductivity enhancements were smaller than those evidenced in the previously-cited works. Conversely, in a report on conducting PANI pellets subjected to up to $400 \mathrm{kGy}$ dose, no increase in conductivity was detected in the 0-200 kGy dose range, with a less than $25 \%$ increase in the 300 $400 \mathrm{kGy}$ range [43]. The increase in conductivity was consistent with the increase in spin or radical concentration detected by Electron Paramagnetic Resonance (EPR) measurements. These and other defects are capable of acting as charge carriers throughout ICPs polymer chains [44-46], thus, explaining the higher conductivity observed.

\subsection{FTIR analysis}

FTIR characterization of PHB and PANI-HCl, nanocomposites II (before and after irradiation at $25 \mathrm{kGy}$ ) and nanocomposite IV are presented in Figure 3. PHB spectrum (Figure 3a) exhibits $\mathrm{C}=\mathrm{O}$ stretching at $1724 \mathrm{~cm}^{-1}$ and $\mathrm{C}-\mathrm{O}$ stretching at $1283 \mathrm{~cm}^{-1}$. These results are in good agreement with previous reports on FTIR data of PHB [47]. PANI-HCl spectrum (Figure $3 \mathrm{~b}$ ) exhibits band at $\sim 1573 \mathrm{~cm}^{-1}$ attributed to $\mathrm{C}=\mathrm{N}$ stretching of the

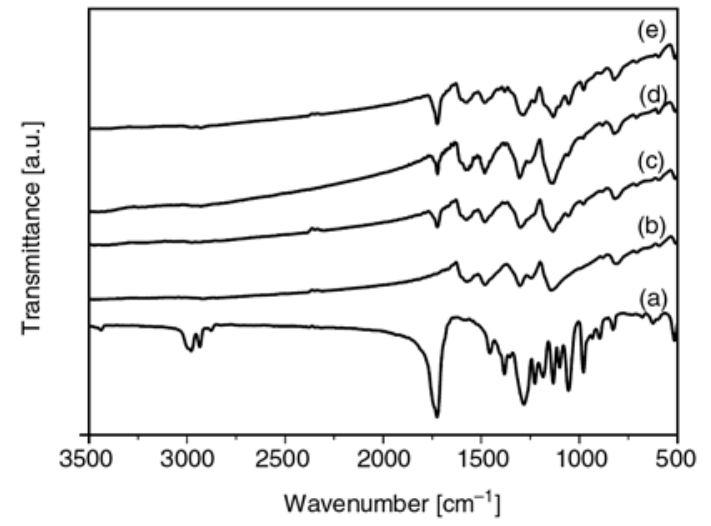

Figure 3. FTIR spectra of (a) PHB, (b) PANI HCl, (c) nanocomposite IV, (d) non-irradiated nanocomposite II and (e) nanocomposite II irradiated at $25 \mathrm{kGy}$

quinoid diimine unit $(\mathrm{N}=\mathrm{Q}=\mathrm{N}) . \mathrm{C}-\mathrm{C}$ aromatic ring stretching of the benzenoid diamine unit $(\mathrm{N}-\mathrm{B}-\mathrm{N})$ appears at $1481 \mathrm{~cm}^{-1}$ [48]. Based on previous reports, the very close intensities presented by these two bands identify the emeraldine oxidation state of PANI [49]. The $\sim 1140 \mathrm{~cm}^{-1}$ band is a vibrational mode of $\mathrm{B}-\mathrm{NH}^{+}=\mathrm{Q}$ or $\mathrm{B}-\mathrm{NH}^{+}=\mathrm{B}$. Both bands are related to the doping level of PANI-HCl and can be used as a comparison of PANI doping process when formed in situ in the PHB matrix. Nanocomposites II and IV spectra (Figures 3c-3e) exhibit the same major bands present in PHB and PANI-HCl spectrum. Only marginal shifts were detected in these nanocomposites, thus intermolecular interactions between the two components are negligible, e.g., $n-\pi$ interactions of the unshared pair of electron of the carbonyl group in PHB and the $\pi$-electrons of the aromatic ring of PANI-HCl are not as perceptible in these composites, as they are in blends of other carbonyl/aromatic polymers [50, 51]. Comparison between the changing in intensity of $\sim 1303$ and $\sim 1140 \mathrm{~cm}^{-1}$ bands shows that doping level of PANI $\mathrm{HCl}$ in these nanocomposites are similar to the doping level of the product obtained in the absence of PHB, even in the gamma-irradiated nanocomposite II (Figure 3e).

\subsection{XRD analysis}

$\mathrm{X}$-ray diffractogram of PHB is shown in Figure 4a. Diffraction peaks in $2 \theta=13.4,16.8,20,22.2$ and $25.5^{\circ}$ have a similar pattern when compared with previous crystallographic data for this material [52]. The degree of crystallinity calculated through Ruland's method considers the total area of the diffracted peaks subtracted of the amorphous halo 


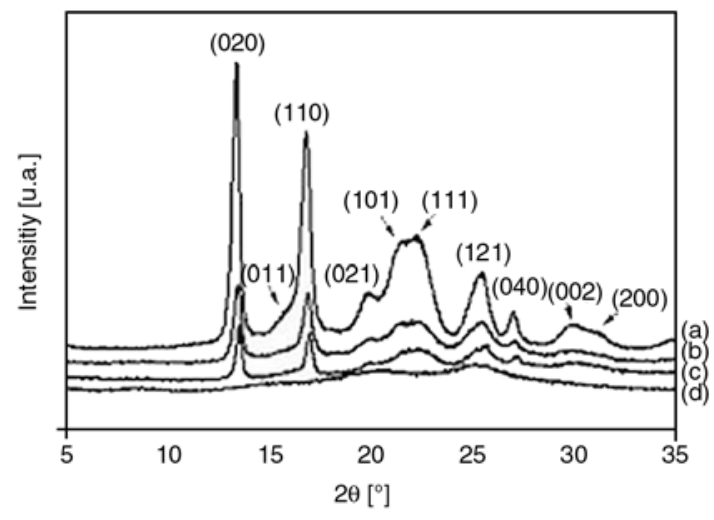

Figure 4. X-ray diffractograms of (a) PHB, (b) nanocomposite II (28\% of PANI), (c) nanocomposite IV (45\% of PANI) and (d) PANI-HCl

[30]. Our results indicated a degree of crystallinity of $53 \%$. This method was previously used to assess the crystalline fraction of as-supplied Brazilian PHB with similar results [53]. PHB/PANI-HCl nanocomposites II and IV diffraction patterns are very close to those exhibited by neat PHB (Figures $4 \mathrm{~b}$ and $4 \mathrm{c}$ ). Nevertheless, it is possible to notice a slight shift to higher angles with the increase of PANI-HCl content, indicating a decrease in the interplanar distance of PHB crystals. In neat PANI$\mathrm{HCl}$ diffractogram, two broad, relatively intense peaks at 20.3 and $25.1^{\circ}$ can be seen, along with two less intense 8.5 and $15.0^{\circ}$ (Figure $4 \mathrm{~d}$ ). This pattern is related to amorphous polyanilines synthesized in the presence of surfactant agents such as SDS and dodecylbenzenesulfonate (SDBS) [54]. When prepared in the absence of such agents or secondary dopants, PANI diffractograms tend to exhibit a single broad weak peak at $2 \theta=24.7^{\circ}[54,55]$. This evidence may indicate a direct inclusion of SDS surfactant used in the emulsion polymerization. Nevertheless, the absence of new crystalline orders corroborates the assumption of poor interaction between PHB and PANI. Since some characteristic diffraction peaks of PANI-HCl partially overlapped those of PHB, crystalline fraction calculations for the nanocomposites did not present consistent results.

\subsection{Biodegradability of PHB/PANI-HCI nanocomposite}

Sturm's test was applied to powders of PHB, PANI$\mathrm{HCl}$ and nanocomposite II. Attempts to promote biodegradation of neat non-irradiated and irradiated PANI-HCl under the tested conditions did not result in any appreciable $\mathrm{CO}_{2}$ production. Changes in PHB biodegradation could be perceived after 10 days. Non-irradiated PHB began to biodegrade releasing lower amounts of $\mathrm{CO}_{2}$ than the other samples and remained this way until the $50^{\text {th }}$ day. Similarly, irradiated PHB started to evolve lower quantities of $\mathrm{CO}_{2}$ than non-irradiated and irradiated nanocomposite II around the $15^{\text {th }}$ day. Nanocomposite II presented similar behavior in non-irradiated and irradiated samples until the $22^{\text {th }}$ day, when the irradiated samples started to exhibit a small decrease in $\mathrm{CO}_{2}$ production. Non-irradiated nanocomposite II maintained higher rates of $\mathrm{CO}_{2}$ release until the $35^{\text {th }}$, when biodegradation of both nanocomposites subsided. Neat PHB samples, in turn, showed rampant increase in $\mathrm{CO}_{2}$ production after the $45^{\text {th }}$ to approximately the $70^{\text {th }}$ day, when both sample media started to show signs of exhaustion. Figure 5a shows $P$ versus time (in days). Figure $5 \mathrm{~b}$ shows the first derivate of $P$ as a function of time $(\mathrm{d} P / \mathrm{d} T)$ and reveals the differences in biodegradation behavior of the studied samples. Nanocomposite samples have a markedly higher biodegradation around the first month of testing (region I) while neat PHB samples showed increased activity starting in the second month of observations (region II), when practically no activity is detected for nanocomposites biodegradation. $\mathrm{CO}_{2}$ accumulated values in region II are higher for irradiated neat PHB as evidenced by higher peaks heights for this sample.

Gamma irradiations affected the studied materials in opposite ways: while irradiated neat $\mathrm{PHB}$ reached higher values of accumulated $\mathrm{CO}_{2}$ production than non-irradiated neat PHB samples, irradiated nanocomposite materials showed lower $\mathrm{CO}_{2}$ accumulation values. Irradiation with gamma rays provokes main chain scissions in PHB structure. In the range of $0-50 \mathrm{kGy}$, the $G$ value (number of scissions per $100 \mathrm{eV}$ of absorbed energy) for BIOCYCLE ${ }^{\circledR}$ is 15.7 [53]. This number of scissions is large enough to reduce the initial molar mass in one order of magnitude after a $25 \mathrm{kGy}$ absorbed dose. Such damage might ease the biodegradation process in neat samples and explain higher $\mathrm{CO}_{2}$ production of these materials. In nanocomposites, radiolysis may not be as remarkable because of a putative radiostabilization of PANI-HCl on PHB matrix, in the same fashion reported for PMMA [19, 20]. Moreover, 

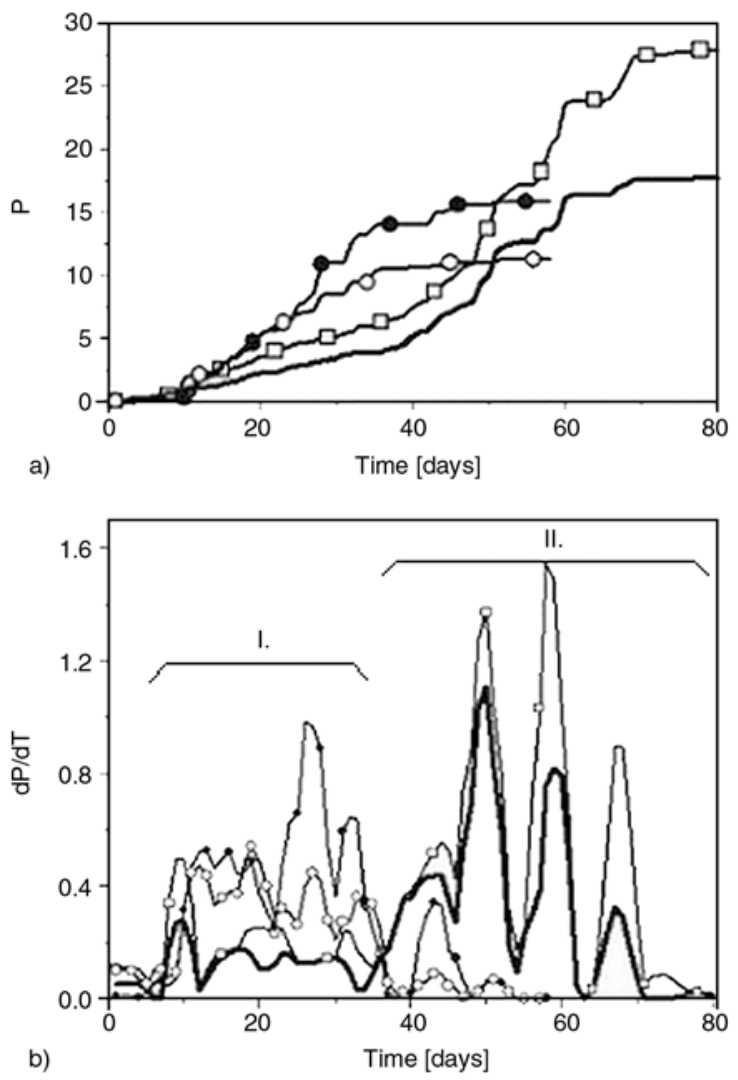

Figure 5. a) Accumulation of $\mathrm{CO}_{2}(P)$ of powder samples of PHB: non-irradiated (-) and irradiated at 25 kGy (-口-); PHB/PANI-HCl (28\% of PANI$\mathrm{HCl})$ non-irradiated $(-\bullet-)$ and irradiated $25 \mathrm{kGy}$ $(-\mathrm{O}-)$. Results are normalized by the $P$ value of the control (inoculant media). Results for the nanocomposite II were also corrected by the percentage of PHB present (72\%). b) First derivative of $P$ in time. Region I shows roughly the first month of observations, when intense peaks associated with nanocomposite biodegradation are present, while neat PHB samples show relatively low $\mathrm{CO}_{2}$ accumulation. Region II delimits approximately the final 40 days of observation, when most of the neat PHB biodegradation occurs.

differences in biodegradation of irradiated neat PHB matrix and PANI nanocomposite may be governed by other factors, as minor alterations caused by irradiation on the surface of the nanocomposite. PANI presents good biocompatibility, either in its emeraldine, nigraniline or leucoemeraldine oxidation states [17]. Moreover, the presence of electrically conducting PANI in polymer nanocomposites with biodegradable polymers allows the fabrication of suitable substrates for tissue engineering, in which electrical stimulation is important to promote cell proliferation and tissue regeneration [28, 29].
PHB/PANI nanocomposites presented in this work have the basic requirements to become one of these multifunctional nanotechnology materials, as they are composed of biocompatible polymers and possess electrical semiconductivity.

Many factors, besides materials biocompatibility may also interfere in substrate performance, e.g. minor synthesis residues such as oligoanilines and toxic unreacted aniline monomers. Thus, further investigations are needed in order to assess the viability of cell attachment and proliferation on the surface of the nanocomposites presented in this work, as well as the influence of electrical stimulation on these and other cytological processes.

\section{Conclusions}

PHB/PANI-HCl nanocomposites were obtained by in situ emulsion polymerization of $\mathrm{HCl}$-doped PANI, with rapid mixing of oxidant, in the presence of dissolved PHB. The nanofiller presented fibrillar or rod-like shape and 70-100 nm in diameter. Conductivity of the nanocomposites were in the range of $10^{-4}$ to $10^{-2} \mathrm{~S} / \mathrm{cm}$. FTIR analysis revealed similar doping levels in PANI-HCl when embedded into the PHB matrix or in the pristine form. XRD patterns of the semicrystalline PHB were present in nanocomposites and PHB diffractograms. PANI$\mathrm{HCl}$ peaks suggested some degree of association with a surfactant agent, SDS, used to promote emulsification. Nevertheless, FTIR and XRD data did not show evidence of significant interactions between PHB and PANI-HCl nanofibers in the composite. Biodegradation experiments showed that PHB biodegradability is enhanced after irradiation at $25 \mathrm{kGy}$. Biodegradation of non-irradiated and irradiated PHB occur more intensely after 30 days, but with a larger accumulated production of $\mathrm{CO}_{2}$ detected for the irradiated samples. PHB/ PANI-HCl nanocomposites present a faster biodegradation than PHB in the first month of measurements. After this period, nanocomposite biodegradation subsided. Irradiated samples exhibited a decrease in the biodegradation process of the nanocomposite after the $22^{\text {th }}$ day of testing. Therefore, accumulated $\mathrm{CO}_{2}$ production is higher in the non-irradiated samples. Neat PANI-HCl biodegradation is negligible when compared to PHB biodegradability in an 80 day timeframe. 


\section{Acknowledgements}

The authors acknowledge The Brazilian Research Council (CNPq) for financial support and PHB Industrial S. A. for kindly supplying BIOCYCLE ${ }^{\circledR}$ samples.

\section{References}

[1] Jayasekara R., Harding I., Bowater I., Lonergan G.: Biodegradability of a selected range of polymers and polymer blends and standard methods for assessment of biodegradation. Journal of Polymers and the Environment, 13, 231-251 (2005).

DOI: $10.1007 / \mathrm{s} 10924-005-4758-2$

[2] Chen G-Q., Wu Q.: The application of polyhydroxyalkanoates as tissue engineering materials. Biomaterials, 26, 6565-6578 (2005).

DOI: 10.1016/j.biomaterials.2005.04.036

[3] Piskin E., Bölgen N., Egri S., Isoglu I. A.: Electrospun matrices made of poly ( $\alpha$-hydroxy acids) for medical use. Nanomedicine, 2, 441-457 (2007).

DOI: $10.2217 / 17435889.2 .4 .441$

[4] Müller H-M., Seebach D.: Poly(hydroxyalkanoates): A fifth class of physiologically important organic biopolymers? Angewandte Chemie International Edition, 32, 477-502 (1993).

DOI: 10.1002 /anie.199304771

[5] Gursel I., Yagmurlu F., Korkusuz F., Hasirci V.: In vitro antibiotic release from poly(3-hydroxybutyrateco-3-hydroxyvalerate) rods. Journal of Microencapsulation, 19, 153-164 (2002).

DOI: $10.1080 / 02652040110065413$

[6] Khang G., Kim S. W., Cho J. C., Rhee J. M., Yoon S. C., Lee H. B.: Preparation and characterization of poly(3-hydroxybutyrate-co-3-hydroxyvalerate) microspheres for the sustained release of 5-fluorouracil. BioMedical Materials and Engineering, 11, 89-103 (2001).

[7] Sudesh K., Abe H., Doi Y.: Synthesis, structure and properties of polyhydroxyalkanoates: Biological polyesters. Progress in Polymer Science, 25, 1503-1555 (2000).

DOI: 10.1016/S0079-6700(00)00035-6

[8] Bordes P., Pollet E., Bourbigot S., Avérous L.: Structure and properties of PHA/clay nano-biocomposites prepared by melt intercalation. Macromolecular Chemistry and Physics, 209, 1473-1484 (2008).

DOI: $10.1002 / \mathrm{macp} .200800022$

[9] Choi W. M., Kim T. W., Park O. O., Chang Y. K., Lee J.W.: Preparation and characterization of poly(hydroxybutyrate-co-hydroxyvalerate)-organoclay nanocomposites. Journal of the Applied Polymer Science, 90, 525-529 (2003).

DOI: $10.1002 / a p p .12702$
[10] Lai M., Li J., Yang J., Liu J., Tong X., Cheng H.: The morphology and thermal properties of multi-walled carbon nanotube and poly(hydroxybutyrate-co-hydroxyvalerate) composite. Polymer International, 53, 1479-1484 (2004).

DOI: $10.1002 /$ pi.1566

[11] Stafström S., Brédas J. L., Epstein A. J., Woo H. S., Tanner D. B., Huang W. S. MacDiarmid A. G.: Polaron lattice in highly conducting polyaniline: Theoretical and optical studies. Physical Review Letters, 59, 1464-1467 (1987). DOI: $10.1103 /$ PhysRevLett.59.1464

[12] Lee M. H., Speyer G., Sankey O. F.: Theory of electron transport through single molecules of polyaniline. Journal of Physics: Condensed Matter, 19, 215204/1215204/16 (2007). DOI: $10.1088 / 0953-8984 / 19 / 21 / 215204$

[13] Cottevieille D., Le Méhauté A., Challioui C., Mirebeau P., Demay J. N.: Industrial applications of polyaniline. Synthetic Metals, 101, 703-704 (1999).

DOI: 10.1016/S0379-6779(98)01093-5

[14] Dutta D., Sarma T. K., Chowdhury D., Chattopadhyay A.: A polyaniline-containing filter paper that acts as a sensor, acid, base, and endpoint indicator and also filters acids and bases. Journal of Colloid and Interface Science, 283, 153-159 (2005).

DOI: $10.1016 /$ j.jcis.2004.08.051

[15] Pron A., Rannou P.: Processible conjugated polymers: From organic semiconductors to organic metals and superconductors. Progress in Polymer Science, 27, 135-190 (2002).

DOI: $10.1016 / \mathrm{S} 0079-6700(01) 00043-0$

[16] Chiang J. C., MacDiarmid A. G.: 'Polyaniline': Protonic acid doping of the emeraldine form to the metallic regime. Synthetic Metals, 13, 193-205 (1986). DOI: 10.1016/0379-6779(86)90070-6

[17] Wang C. H., Dong Y. Q., Sengothi K., Tan K. L., Kang E. T.: In-vivo tissue response to polyaniline. Synthetic Metals, 102, 1313-1314 (1999). DOI: 10.1016/S0379-6779(98)01006-6

[18] Kang E. T., Neoh K. G., Tan K. L.: Polyaniline: A polymer with many interesting intrinsic redox states. Progress in Polymer Science, 23, 277-324 (1998). DOI: 10.1016/S0079-6700(97)00030-0

[19] Araujo P. L. B., Santos R. F. S., Araujo E. S.: Polyaniline nanofibers as a new gamma radiation stabilizer agent for PMMA. Express Polymer Letters, 1, 385390 (2007).

DOI: $10.3144 /$ expresspolymlett.2007.54

[20] Araujo P. L. B., Aquino K. A. S., Araujo E. S.: Effects of gamma irradiation on PMMA/polyaniline nanofibre composites. International Journal of Low Radiation, 4 , 149-160 (2007).

DOI: 10.1504/IJLR.2007.015439 
[21] Huang J., Kaner R. B.: The intrinsic nanofibrillar morphology of polyaniline. Chemical Communications, 367-376 (2006).

DOI: $10.1039 / \mathrm{b} 510956 \mathrm{f}$

[22] Chien J. C. W., Yamashita Y., Hirsch J. A., Fan J. L., Schen M. A., Karasz F. E.: Resolution of controversy concerning the morphology of polyacetylene. Nature, 299, 608-611 (1982).

DOI: $10.1038 / 299608 \mathrm{a} 0$

[23] Ruckenstein E., Yang S.: An emulsion pathway to electrically conductive polyaniline-polystyrene composites. Synthetic Metals, 53, 283-292 (1993). DOI: 10.1016/0379-6779(93)91097-L

[24] Yang S., Ruckenstein E.: Processable conductive composites of polyaniline/poly(alkyl methacrylate) prepared via an emulsion method. Synthetic Metals, 59, 1-12 (1993). DOI: 10.1016/0379-6779(93)91152-R

[25] Huang J., Kaner R. B.: Nanofiber formation in the chemical polymerization of aniline: A mechanistic study. Angewandte Chemie, 113, 5941-5945 (2004). DOI: 10.1002/ange.200460616

[26] Ali M. A., Saion E., Yahya N., Kassim A., Dahlan K. M., Hashim S.: Synthesis of conducting polyaniline nanocomposites by radiation doping. Journal of Engineering Science and Technology, 2, 111-118 (2007).

[27] Fryczkowski R., Kowalczyk T.: Nanofibres from polyaniline/polyhydroxybutyrate blends. Synthetic Metals, 159, 21-22 (2009).

DOI: 10.1016/j.synthmet.2009.09.008

[28] Ghasemi-Mobarakeh L., Prabhakaran M. P., Morshed M., Nasr-Esfahani M. H., Ramakrishna S.: Electrical stimulation of nerve cells using conductive nanofibrous scaffolds for nerve tissue engineering. Tissue Engineering Part A, 15, 3605-3619 (2009). DOI: $10.1089 /$ ten.tea.2008.0689

[29] McKeon K. D., Lewis A., Freeman J. W.: Electrospun poly(D,L-lactide) and polyaniline scaffold characterization. Journal of Applied Polymer Science, 115, 1566-1572 (2009).

DOI: 10.1002 /app.31296

[30] Ruland W.: X-ray determination of crystallinity and diffuse disorder scattering. Acta Crystallographica, 14, 1180-1185 (1961).

DOI: $10.1107 / \mathrm{S} 0365110 \mathrm{X} 61003429$

[31] Calil M. R., Gaboardi F., Guedes C. G. F., Rosa D. S.: Comparison of the biodegradation of poly( $\varepsilon$-caprolactone), cellulose acetate and their blends by the Sturm test and selected cultured fungi. Polymer Testing, 25, 597-604 (2006).

DOI: $10.1016 /$ j.polymertesting.2006.01.019

[32] ASTM D5338-98.: Standard test method for determining aerobic biodegradation of plastic materials under controlled composting conditions (2003).

[33] Anderson A. J., Dawes, E. A.: Occurrence, metabolism, metabolic role, and industrial uses of bacterial polyhydroxyalkanoates. Microbiological Reviews, 54, 450-472 (1990).
[34] Huang J. X., Virji S., Weiller B. H., Kaner R. B.: Polyaniline nanofibers: Facile synthesis and chemical sensors. Journal of the American Chemical Society, 125, 314-315 (2003).

DOI: $10.1021 / \mathrm{ja} 028371 \mathrm{y}$

[35] Hopkins A. R., Lipeles R. A., Kao W. H.: Electrically conducting polyaniline microtube blends. Thin Solid Films, 447-448, 474-480 (2004).

DOI: $10.1016 /$ j.tsf.2003.07.010

[36] Carswell A. D. W., O'Rear E. A., Grady B. P.: Adsorbed surfactants as templates for the synthesis of morphologically controlled polyaniline and polypyrrole nanostructures on flat surfaces: From spheres to wires to flat films. Journal of the American Chemical Society, 125, 14793-14800 (2003).

DOI: $10.1021 / \mathrm{ja} 0365983$

[37] Pan W., Yang S. L., Li G., Jiang J. M.: Electrical and structural analysis of conductive polyaniline/polyacrylonitrile composites. European Polymer Journal, 41, 2127-2133 (2005).

DOI: 10.1016/j.eurpolymj.2005.04.003

[38] Sevil U. A., Güven O., Süzer S.: Spectroscopic investigation of onset and enhancement of electrical conductivity in PVC/PANI composites and blends by $\gamma$-ray or UV irradiation. Journal of Physical Chemistry B, 102, 3902-3905 (1998).

DOI: $10.1021 / \mathrm{jp} 980369 \mathrm{z}$

[39] Sevil U. A., Güven O., Birer O., Şüzer S.: Doping of 2Cl-PANI/PVC films by exposure to UV, $\gamma$-rays and e-beams. Synthetic Metals, 110, 175-179 (2000). DOI: 10.1016/S0379-6779(99)00266-0

[40] Sevil U. A., Güven O., Kovács A., Slezsák I.: Gamma and electron dose response of the electrical conductivity of polyaniline based polymer composites. Radiation Physics and Chemistry, 67, 575-580 (2003). DOI: 10.1016/S0969-806X(03)00108-7

[41] Bodugöz H., Güven O.: Radiation induced dehydrochlorination as an in-situ doping technique for enhancement of the conductivity of polyaniline blends. Nuclear Instruments and Methods in Physics Research Section B: Beam Interactions with Materials and Atoms, 236, 153-159 (2005).

DOI: $10.1016 /$ j.nimb.2005.04.009

[42] Azevedo W. M., de Costa Lima A. P., Araujo E. S.: Radiation induced effects on electrical properties of polyaniline. Radiation Protection Dosimetry, 84, 7781 (1999).

[43] Yao Q., Liu L., Li C.: Radiation induced variations of electrical conductivity in polyaniline. Chinese Physics Letters, 11, 113-115 (1994). DOI: 10.1088/0256-307X/11/2/014

[44] Fesser K.: Interchain coupling in trans-polyacetylene. Physical Review B, 40, 1962-1965 (1989). DOI: 10.1103/PhysRevB.40.1962

[45] Krinichnyi V. I., Chemerisov S. D., Lebedev Ya. S.: EPR and charge-transport studies on polyaniline. Physical Review B, 55, 16233-16244 (1997). DOI: 10.1103/PhysRevB.55.16233 
[46] Kivelson S.: Frustration, fractional charge, and soliton mobilities: The effect of interchain coupling on solitons in charge-density waves. Physical Review B, 26, 7093-7096 (1982).

DOI: 10.1103/PhysRevB.26.7093

[47] Xu J., Guo B-H., Yang R., Wu Q., Chen G. Q., Zhang Z-M.: In situ FTIR study on melting and crystallization of polyhydroxyalkanoates. Polymer, 43, 68936899 (2002).

DOI: 10.1016/S0032-3861(02)00615-8

[48] Tang J., Jing X., Wang B., Wang F.: Infrared spectra of soluble polyaniline. Synthetic Metals, 24, 231-238 (1988).

DOI: 10.1016/0379-6779(88)90261-5

[49] Abdiryim T., Zhang X-G., Jamal R.: Comparative studies of solid-state synthesized polyaniline doped with inorganic acids. Materials Chemistry and Physics, 90, 367-372 (2005).

DOI: $10.1016 /$ j.matchemphys.2004.10.036

[50] Koenig J. L.: Spectroscopy of polymers. Elsevier, Amsterdam (1999).
[51] Mohamed A., Gordon S. H., Biresaw G.: Polycaprolactone/polystyrene bioblends characterized by thermogravimetry, modulated differential scanning calorimetry and infrared photoacoustic spectroscopy. Polymer Degradation and Stability, 92, 1177-1185 (2007).

DOI: $10.1016 /$ j.polymdegradstab.2007.04.012

[52] Ikejima T., Inoue Y.: Crystallization behavior and environmental biodegradability of the blend films of poly(3-hydroxybutyric acid) with chitin and chitosan. Carbohydrate Polymers, 41, 351-356 (2000).

DOI: 10.1016/S0144-8617(99)00105-8

[53] Oliveira L. M., Araujo E. S., Guedes S. M. L.: Gamma irradiation effects on poly(hydroxybutyrate). Polymer Degradation and Stability, 91, 2157-2162 (2006).

DOI: $10.1016 /$ j.polymdegradstab.2006.01.008

[54] Cai L-T., Yao S-B., Zhou S-M.: Improved conductivity and electrical properties of polyaniline in the presence of rare-earth cations and magnetic field. Synthetic Metals, 88, 205-212 (1997).

DOI: $10.1016 / \mathrm{S} 0379-6779(97) 03851-4$

[55] MacDiarmid A. G., Epstein A. J.: The concept of secondary doping as applied to polyaniline. Synthetic Metals, 65, 103-116 (1994).

DOI: 10.1016/0379-6779(94)90171-6 